\section{Aplicativo móvel de apoio à aspiração do tubo endotraqueal e de vias aéreas superiores}

\section{Mobile application to support endotracheal tube and upper airway aspiration}

\author{
Geraldo Magela Salome, Gislaine Cristina Martins Rosa
}

\section{RESUMO}

Objetivo: desenvolver um aplicativo multimídia em plataforma móvel para guiar, passo-a-passo, 0 procedimento de aspiração da cânula endotraqueal e das vias aéreas superiores. Método: estudo metodológico. A estrutura do aplicativo foi desenvolvida em 4 etapas: (1) identificação das necessidades do desenvolvimento do aplicativo, visto que alguns profissionais e cuidadores realizam a técnica de aspiração inadequadamente; (2) desenvolvimento do protótipo do aplicativo com base em revisão integrativa da literatura; (3) construção do aplicativo com a elaboração de árvores de decisão, algoritmos, estruturação do banco de dados e desenvolvimento do software; e (4) realização de testes de funcionalidade. Resultados: 0 aplicativo desenvolvido é composto por 81 telas com 52 ilustrações. Os testes de usabilidade, desempenho, compatibilidade e funcional foram realizados em 2 tipos de notebook, 6 tipos de smartphone e 2 tipos de tablete. Cinco problemas foram detectados e corrigidos antes do aplicativo ser disponibilizado ao usuário. 0 aplicativo deu entrada no registro de programa de computador no Instituto Nacional da Propriedade Industrial (processo número BR5120180524354). Conclusão: foi desenvolvido o aplicativo multimídia em plataforma móvel (Asptraqueal-App) para apoio a procedimentos de aspiração do tubo endotraqueal e de vias aéreas superiores, o qual poderá ter grande utilidade para cuidadores e profissionais de saúde.

PALAVRAS-CHAVE: Aplicativos Móveis; Software; Sucção; Traqueostomia; Aplicações da Informática Médica.

\section{ABSTRACT}

Objective: to develop a multimedia application on a mobile platform to guide, step-by-step, the procedure for aspiration of the endotracheal cannula and upper airways. Method: methodological study. The application structure was developed in 4 stages: (1) identification of the needs of the application development, since some professionals and caregivers perform the aspiration technique inappropriately; (2) development of the application prototype based on an integrative literature review; (3) building the application with the elaboration of decision trees, algorithms, structuring the database and software development; and (4) performance of functionality tests. Results: the application developed consists of 81 screens with 52 illustrations. The usability, performance, compatibility and functional tests were performed on 2 types of notebook, 6 types of smartphone and 2 types of tablet. Five problems were detected and corrected before the application was made available to the user. The application was registered in the computer program registration at the National Institute of Industrial Property (process number BR512018052435-4). Conclusion: the multimedia application was developed on a mobile platform (Asptraqueal-App) to support endotracheal tube and upper airway aspiration procedures, which may be of great use to caregivers and health professionals.

KEYWORDS: Mobile Applications; Software; Suction; Tracheostomy; Medical Informatics Applications. 


\section{INTRODUÇÃO}

A manutenção da perviabilidade das vias aéreas é o principal objetivo da assistência prestada aos pacientes entubados e traqueostomizados. A presença do tubo artificial impede que o paciente faça o mecanismo normal de limpeza das vias aéreas ao tossir. A tosse é conhecida como um reflexo natural do corpo para eliminar qualquer irritação pulmonar ${ }^{1}$. O acúmulo de secreções é inevitável, pois a cânula endotraqueal impede que os mecanismos de defesa das vias aéreas superiores, como filtração, umidificação e aquecimento do ar sejam utilizados normalmente, e prejudica também o reflexo de tosse ${ }^{2-3}$.

A aspiração traqueobrônquica consiste na introdução de uma sonda nas vias áreas do paciente para extrair as secreções. A sonda de aspiração deve ser conectada a um aspirador com pressão de sucção ou pressão negativa e a um cateter de aspiração, sendo o seu calibre apropriado quando a relação de 1/3 do tubo traqueal é observada para que haja movimento de fluídos e gases, promovendo, assim, a oxigenação adequada e impedindo a obstrução das vias respiratórias ${ }^{4-6}$.

A utilização de tecnologias computacionais na área educacional e da saúde vem inovando a relação ensinoaprendizagem e teoria-prática na assistência à medida que são adaptadas às necessidades da assistência segura ao paciente e dos modelos educacionais contemporâneos. Os profissionais da área de saúde acompanham essa inovação, e por meio de experiências com a utilização de ambientes virtuais de aprendizagem, têm demonstrado que a interatividade favorece o processo de aprendizado e melhora a assistência segura, sem danos para o paciente ${ }^{7-11}$. A utilização de aplicativos assistenciais, como planejamento do cuidado informatizado, agiliza as atividades de coleta, registro, armazenamento, manipulação e recuperação de dados dos pacientes. Além da facilidade de acesso aos dados, o aplicativo possibilita instrumentalização administrativa e auxilia na tomada de decisões ${ }^{9,12-15}$.

A construção de um aplicativo para aparelhos móveis oferece aos profissionais e cuidadores um meio de consulta rápida, facilmente transportado para os diversos cenários de práticas em estabelecimentos de saúde. Através do acesso a este tipo de plataforma, o profissional ou o cuidador conseguirá sanar algumas dúvidas, e com mais autonomia, se sentirá mais seguro em executar vários procedimentos, oferecendo segurança e compreendendo melhor o que aquele resultado significa em termos de evolução clínica para o paciente 5,7,10,12,16-17.

Poucos são os estudos desenvolvidos para padronizar as recomendações da técnica de aspiração traqueobrônquica das vias aéreas, tornando-se importante a busca da melhoria da aplicação dessa técnica para prevenir complicações e promover o bem-estar e conforto do paciente. Destaca-se a relevância do desenvolvimento de um aplicativo móvel que ofereça ao profissional e ao cuidador um meio de rápida consulta para que ele possa prestar um cuidado com segurança minimizando os riscos durante a realização deste procedimento. 
Assim, o objetivo do presente estudo busca desenvolver um aplicativo multimídia em plataforma móvel para guiar, passo-a-passo, o procedimento de aspiração da cânula endotraqueal e vias aéreas superiores.

\section{MÉTODO}

Trata-se de uma pesquisa metodológica com foco no desenvolvimento, avaliação e aperfeiçoamento de instrumentos e estratégias metodológicas e tecnológicas. O estudo foi aprovado pelo Comitê de Ética em Pesquisa da Faculdade de Ciências Médicas Dr. José Antônio Garcia Coutinho da Universidade do Vale do Sapucaí (UNIVAS), sob o Parecer Consubstanciado 2.889.820, e realizado entre maio de 2017 e setembro de 2018.

A estrutura do aplicativo foi desenvolvida em 4 etapas: (1) concepção e identificação das necessidades do desenvolvimento do aplicativo; (2) elaboração do protótipo do aplicativo com base em revisão da literatura; (3) construção do software; e (4) realização de testes de funcionalidade.

Na etapa 1, buscou-se identificar a necessidade do desenvolvimento do aplicativo, visto que a experiência dos autores na prática clínica voltada à assistência aos pacientes com necessidade de aspiração das vias aéreas indicava que vários profissionais e cuidadores têm dificuldade em realizar o procedimento de forma correta por falta de conhecimento da técnica de aspiração, levando a riscos, danos e falta de segurança ao paciente.

A etapa 2 consistiu na revisão integrativa da literatura e escolha de artigos para a construção dos algoritmos e da árvore de decisão do aplicativo. Uma busca foi realizada junto às bases de dados em Ciências da Saúde, incluindo os bancos de dados Biblioteca Cochrane, Scientific Eletronic Library Online (SciELO), Literatura Latino Americana e do Caribe em Ciência da Saúde (LILACS), e Sistema Online de Busca e Análise de Literatura Médica (MEDLINE) utilizandose o termo "aspiração endotraqueal". Foram avaliados artigos nos idiomas português, espanhol e inglês.

Os critérios de inclusão para seleção das publicações foram: estudos primários que tivessem ligação direta a temática; estar disponível na íntegra, e sem delimitação temporal proposta, pois a intenção era compilar todos os estudos que atendessem aos critérios estabelecidos. Foram excluídos capítulos de livros, teses, dissertações, monografias, relatórios técnicos, trabalhos de referência, e artigos que após leitura do resumo, não convergiam com o objeto de estudo proposto, além das publicações repetidas em bases de dados e biblioteca virtual.

Na etapa 3, primeiramente foi elaborado um protótipo do aplicativo em forma de algoritmo (Figura 1), descrevendo os passos da funcionalidade do aplicativo. 
Figura 1 - Algoritmo para orientar a construção do aplicativo multimídia em plataforma móvel para apoio à aspiração do tubo endotraqueal e vias aéreas superiores. Pouso Alegre, MG, Brasil, 2019.

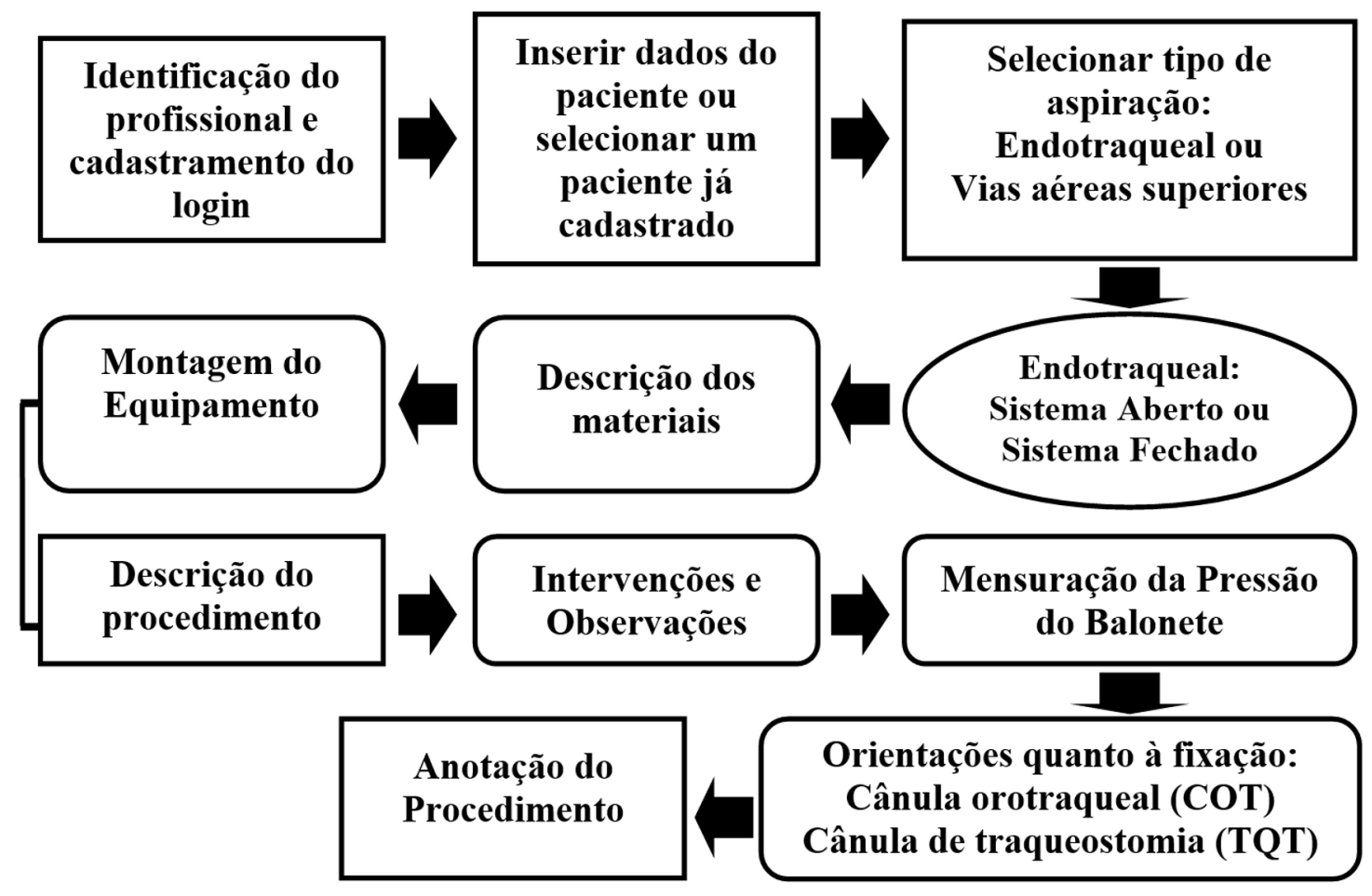

A construção do aplicativo foi realizada com a ajuda de um analista de sistemas devido à especificidade do conhecimento computacional exigido. Foi utilizado o Modelo Incremental de Ciclo de Vida para desenvolvimento de softwares, seguindo o protocolo PRAXIS como base para o processo de engenharia de software ${ }^{8-9,11,13,18-19}$. A plataforma Android e a linguagem JAVA foram usadas para o desenvolvimento do protótipo.

Para construção do aplicativo, foi necessário criar o algoritmo e a árvore de decisão para a estruturação do banco de dados e desenvolvimento do software. $\mathrm{O}$ algoritmo foi estruturado com base na revisão da literatura. $\mathrm{A}$ árvore de decisão foi estruturada com base na revisão da literatura e no algoritmo desenvolvido, como mostrado na Figura 2. 
Figura 2 - Árvore de decisão para construção do aplicativo multimídia em plataforma móvel para apoio à aspiração do tubo endotraqueal e vias aéreas superiores. Pouso Alegre, MG, Brasil, 2019.

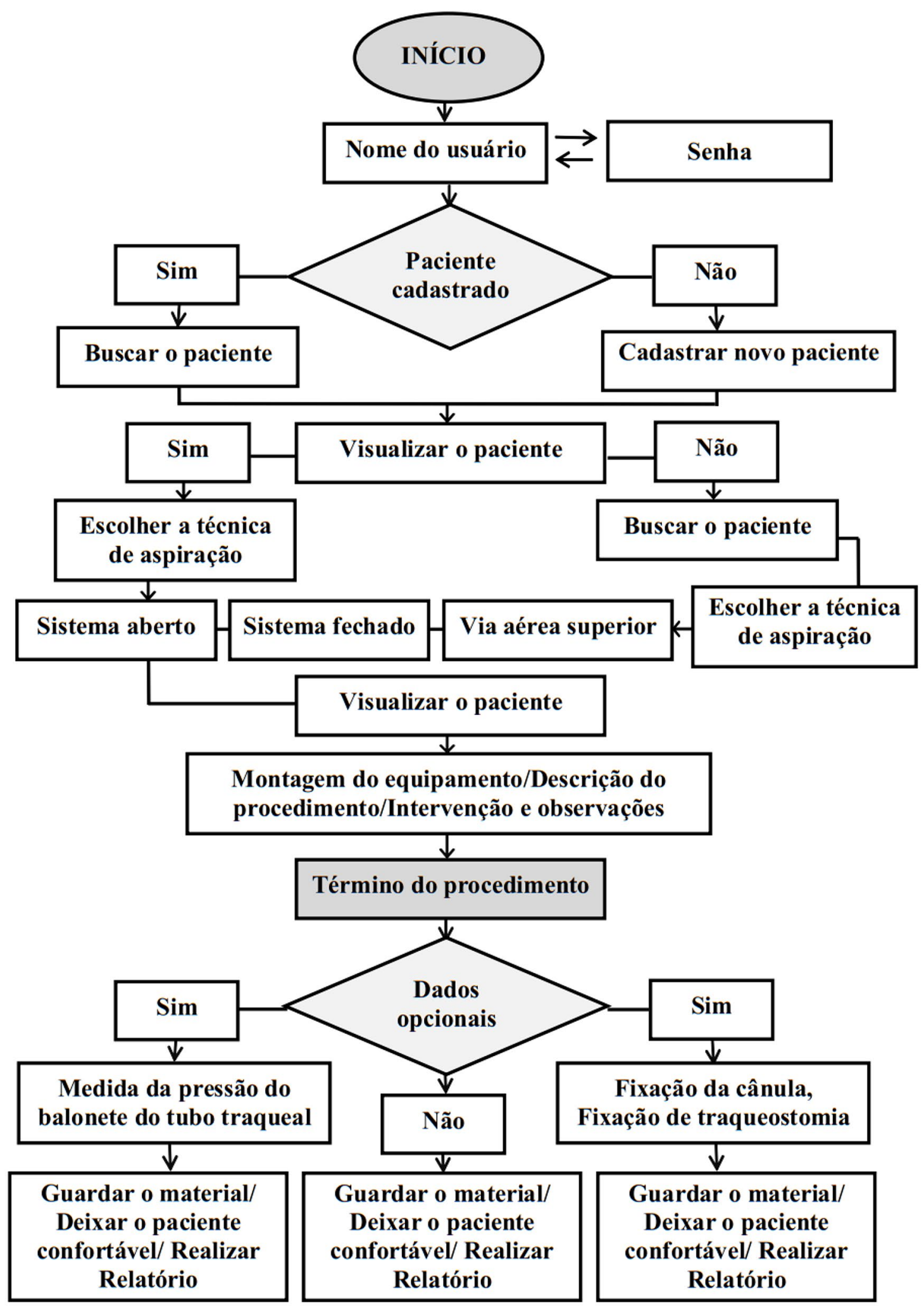

Na etapa 4 foram realizados testes de funcionalidade (usabilidade, desempenho, compatibilidade e funcional).

O teste de usabilidade é utilizado para verificar se o usuário pode utilizar intuitivamente o software da tela inicial 
até o resultado final. Os pesquisadores utilizaram o software cinco vezes, realizando cadastros do paciente, procedimento de aspiração endotraqueal (sistema fechado e sistema aberto) e de vias aéreas superiores, medidas do controle da pressão do balonete, da troca da fixação da cânula ou traqueostomia, e anotações.

O teste de desempenho foi aplicado para avaliar a capacidade de resposta após cada comando efetuado. Assim, foi verificado, pelo analista de sistemas e pesquisadores, o tempo de inicialização, mudança das telas e finalização do software, levando-se em consideração cada uma das telas.

O teste de compatibilidade com o referencial teórico foi dividido em dois estágios: primeiro foram verificadas as informações a nível semântico e sintático do conteúdo do software; e no segundo estágio, foi utilizado o teste funcional ou caixa-preta para testar o sistema, o qual foi conduzido pelo analista de sistemas.

Para o teste funcional do software foram escolhidos dispositivos do tipo móvel que utilizassem a plataforma Android e com Wi-Fi disponível para acesso à rede sem fio, onde foram realizados os testes de usabilidade e compatibilidade.

Todo o processo de testes foi conduzido pelos autores e pelo analista de sistemas. O aplicativo só foi disponibilizado ao usuário após a avaliação da funcionalidade e correções dos problemas detectados.

\section{RESULTADO}

O aplicativo multimídia em plataforma móvel, denominado "Asptraqueal-App", tem 81 telas e 52 figuras descrevendo as técnicas da aspiração do tubo endotraqueal e das vias aéreas superiores. 0 aplicativo deu entrada no registro de programa de computador no Instituto Nacional da Propriedade Industrial do Ministério do Desenvolvimento, Indústria e Comércio Exterior (processo número BR512018052435-4) e está disponível gratuitamente no http://www. aspiapp.com.br/.

A abertura do aplicativo é definida pela logomarca do Mestrado Profissional em Ciências Aplicadas a Saúde da UNIVAS e nome dos autores. Exemplos de telas do aplicativo são apresentados nas Figuras 3 e 4. 
Figura 3 - Exemplos de telas do aplicativo.
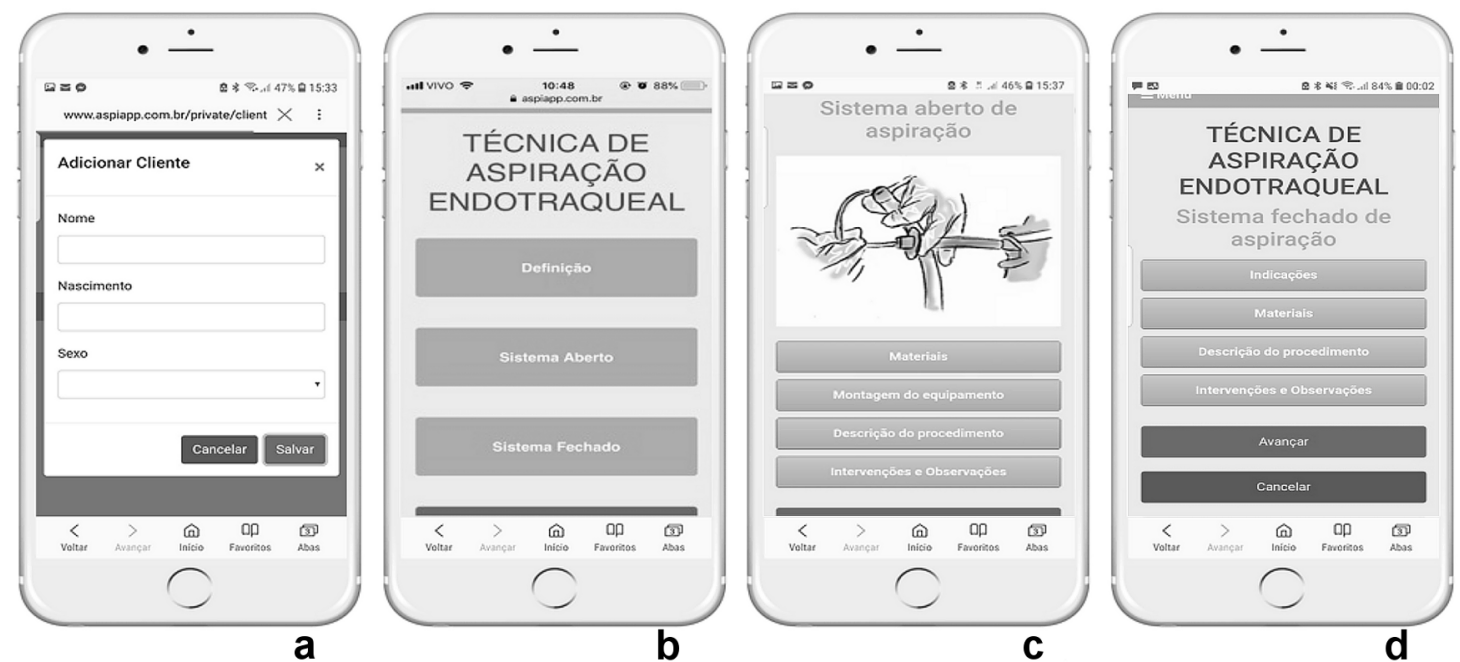

Legenda: (a) Cadastro do paciente; Menus de (b) Técnicas de aspiração endotraqueal, (c) Sistema aberto de aspiração, (d) Sistema fechado de aspiração. Pouso Alegre, MG, Brasil, 2019.

Figura 4 - Exemplos de telas do aplicativo.
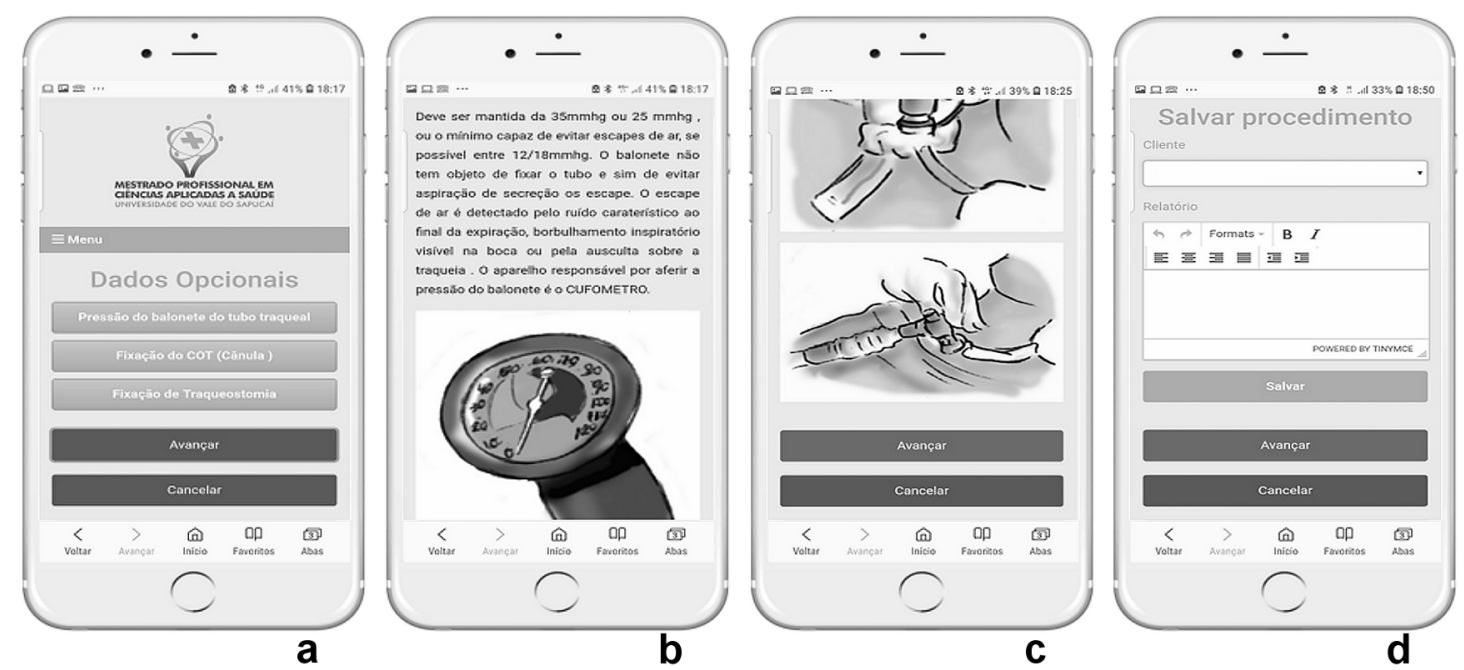

Legenda: (a) Dados opcionais; (b) Texto parcial sobre mensuração da pressão do balonete e ilustração; (c) llustrações da técnica de fixação da traqueostomia; (d) Tela para a realização de anotações. COT, Cânula Orotraqueal. Pouso Alegre, MG, Brasil, 2019.

Após clicar no ícone Entrar, o usuário poderá digitar o nome do paciente cadastrado e inciar o procedimento. Caso o paciente não esteja cadastrado, o usuário deverá realizar o cadastro, digitando o nome, data de nascimento e sexo do cliente (Figura 3a). O usuário deverá então clicar Entrar, abrindo a tela com as descrições das técnicas de aspiração endotraqueal e aspiração das vias aéreas superiores, onde o usuário deverá escolher a técnica que ele irá realizar.

Na tela Técnicas de aspiração endotraqueal (Figura 3b) o usuário pode escolher o tipo de técnica (sistema aberto ou fechado) e, em seguida, terá a opção de escolher o tipo de material, montagem do equipamento para aspiração, 
descrição do procedimento e intervenções (Figuras 3c e 3d). 0 procedimento do sistema aberto é composto por 25 telas e o procedimento do sistema fechado é formado por 15 telas.

Após realizar os procedimentos, o usuário pode optar por Finalizar ou Avançar. A opção Avançar leva à tela com Dados opcionais (Figura 4a), onde a técnica da mensuração da pressão do balonete do tubo traqueal ou da traqueostomia (composta por 8 telas) pode ser acessada (Figura 4b). O usuário também pode verificar as técnicas de fixação da Cânula Orotraqueal (COT) ou da traqueostomia (Figura 4c).

Após finalizar os procedimentos da técnica de aspiração endotraqueal, o usuário pode optar pela realização do procedimento de aspiração de secreções das vias aéreas superiores (composto por 8 telas). Quando o procedimento estiver concluído, o profissional deverá avançar para acessar uma tela onde poderá fazer anotações e rever todas as anotações anteriores realizadas relacionadas ao paciente (Figura 4d).

\section{DISCUSSÃO}

Os aplicativos móveis ganharam força na área da saúde nos últimos anos. Uma imensa variedade de aplicativos está disponível online, incluindo desde sistemas fitness ao monitoramento e controle das mais diversas doenças. Quando bem elaborados, são ferramentas didáticas que podem trazer benefícios para pacientes e profissionais de saúde ${ }^{20-22}$. Vários estudos similares sobre o uso de aplicativos móveis voltados para prática em saúde destacam a importância do investimento nesse campo de pesquisa e na melhoria da assistência sem riscos, danos e eventos adversos ${ }^{8-13}$.

O aplicativo desenvolvido nesse estudo buscou atender às necessidades dos profissionais de saúde e cuidadores, oferecendo informações sobre condutas preventivas, indicações de materiais utilizados, descrição da técnica de aspiração endotraqueal e de vias aéreas superiores, mensuração da pressão do balonete do tubo endotraqueal ou da traqueostomia, e fixação do tubo orotraqueal e da traqueostomia, buscando esclarecer dúvidas.

O uso de aplicativos como ferramenta de condutas terapêuticas, preventivas, diagnósticas e de ensino na área de saúde é bastante inovador e capaz de gerar interesse e motivação pelo aprendizado, já que os aparelhos móveis que hospedam esses aplicativos são utilizados por $45 \%$ a $85 \%$ dos profissionais de saúde ou cuidadores, sendo consultados mais que livros e revistas ${ }^{23}$. Um aplicativo da área da saúde deve contribuir no gerenciamento da assistência, indicar medidas preventivas e auxiliar na formulação do diagnóstico, fornecendo subsídios para uma avaliação clínica dos fatores de risco de desenvolvimento de uma doença ou complicação ${ }^{11-13}$.

O Asptraqueal-App pode ser considerado uma inovação tecnológica em saúde por ser o primeiro aplicativo móvel produzido no Brasil para apoio na aspiração endotraqueal e de vias aéreas superiores, trazendo benefícios aos profissionais de saúde, cuidadores e pacientes atendidos a nível hospitalar, ambulatorial e domiciliar, e proporcionando 
um cuidado com menores riscos ao paciente. $\mathrm{O}$ aplicativo foi criado após revisão da literatura e submetido a testes de usabilidade, desempenho, compatibilidade e funcional, sendo disponibilizado ao usuário somente após a correção dos defeitos detectados 8 -9,13. Aplicativos construídos com embasamento científico favorecem a execução da técnica correta e de cuidados gerais, registro sistemático e individualizado do cuidado, possibilitando uma assistência segura. O atendimento sistematizado das vias aéreas, bem como a otimização da ventilação e oxigenação minimiza o tempo de hospitalização e permite a análise de custos e benefícios do tratamento utilizado.

O Asptraqual-App permite o acesso rápido às informações das principais diretrizes nacionais e internacionais durante a avaliação clínica através de smartphones e tabletes. Ele auxilia o profissional na coleta de dados, pois fornece um relatório de todos os procedimentos realizados na avaliação e na execução das técnicas, além de recomendações para cada achado clínico. Contribui também na rotina do profissional, aumentando o seu conhecimento cientifico, pois coloca no bolso do profissional uma ferramenta atualizada que o auxilia na prática, no exercício do papel de desenvolver ações para prevenção de complicações, danos e riscos.

\section{CONSIDERAÇÕES FINAIS}

Após revisão integrativa da literatura obtida nas principais bases de dados, desenvolveu-se o aplicativo multimídia em plataforma móvel, denominado Asptraqueal-App, para apoio à aspiração do tubo endotraqueal e de vias aéreas superiores. Esta é uma ferramenta prática para qualificar e guiar o profissional na realização da aspiração da cânula endotraqueal e de vias aéreas superiores.

O aplicativo desenvolvido neste estudo tem o potencial de reduzir eventos adversos, proporcionando a prestação do cuidado com o mínimo de risco, danos, e maior segurança e qualidade, porem sua atualização será realizado conforme as nova evidência.

\section{REFERÊNCIAS}

1. Balbino CM, Braz MR, Medeiros JC, Rodrigues LM, Silvino ZR. Evaluation of aspiration technique on the patient with mechanical ventilation performed by nursing. J Nurs UFPE Online. [Internet]. 2016 Dec [cited Sep 10, 2019];10:4797-803. Available from: https://periodicos.ufpe.br/revistas/revistaenfermagem/article/view/11258/12881

2. Ferreira AO, Lima DV, Christovam BP, Silvino ZR. Endotracheal suctioning in intensive care unit: an integrative review. J Nurs UFPE Online. [Internet]. 2013 Jul [cited Sep 10, 2019]:7:4910-7. Available from: https://periodicos. ufpe.br/revistas/revistaenfermagem/article/view/11750/14028 
3. Silva Júnior JG, Silveira JM. Tracheostomy influence on mechanical ventilation time. Rev Amazônia Sci Health. [Internet]. 2017 Jan-Mar [cited Sep 10, 2019];5:35-9. Available from: http://ojs.unirg.edu.br/index.php/2/article/ viewFile/1386/pdf

4. Batista MA, Alcantara EC, Paula LK. Central of mechanical fan: organization, safety and quality. Rev Bras Ter Intensiva. 2007 Oct-Dec;19:450-5. doi: http://dx.doi.org/10.1590/S0103-507X2007000400008

5. Carmo AF, Korinfsky JP, Xavier CC, Mendes RN, Nunes GF, Silva RM. Nursing in ventilatory assistance: analysis of endotracheal aspiration in the intensive care unit. J Nurs UFPE Online. [Internet]. 2013 Dec [cited Sep 10, 2019];7:6800-7. Available from: https://periodicos.ufpe.br/revistas/revistaenfermagem/article/view/12341/15056

6. Gonçalves RL, Tsuzuki LM, Carvalho MG. Endotracheal suctioning in intubated newborns: an integrative literature review. Rev Bras Ter Intensiva. 2015 Jul-Sep;27:284-92. doi: http://dx.doi.org/10.5935/0103-507X.20150048

7. Pereira FG, Silva DV, Sousa LM, Frota NM. Building a digital application for teaching vital signs. Rev Gaucha Enferm. 2016 Jun;37:e59015. doi: http://dx.doi.org/10.1590/1983-1447.2016.02.59015

8. Salomé GM, Bueno JC, Ferreira LM. Multimedia application in a mobile platform for wound treatment using herbal and medicinal plants. J Nurs UFPE Online. [Internet]. 2017 Nov [cited Sep 10, 2019];11:4579-88. Available from: https://periodicos.ufpe.br/revistas/revistaenfermagem/article/view/231197/25190

9. Salomé GM, Ferreira LM. Developing a mobile app for prevention and treatment of pressure injuries. Adv Skin Wound Care. 2018 Feb;31:1-6. doi: http://dx.doi.org/10.1097/01.ASW.0000529693.60680.5e

10. Carvalho MR, Salomé GM, Ferreira LM. Construction and validation of algorithm for treatment of pressure injury. J Nurs UFPE Online. [Internet]. 2017 Oct [cited Sep 10, 2019];11:4171-83. Available from: https://periodicos. ufpe.br/revistas/revistaenfermagem/article/view/231180/25156

11. Cunha DR, Dutra RA, Salomé GM, Ferreira LM. Construction of a multimedia application in a mobile platform for wound treatment with laser therapy. J Nurs UFPE Online. [Internet]. 2018 May [cited Sep 10, 2019];12:680-5. Available from: https://periodicos.ufpe.br/revistas/revistaenfermagem/article/view/230676/28867 
12. Galvão EC, Püschel VA. Multimedia application in mobile platform for teaching the measurement of central venous pressure. Rev Esc Enferm USP. 2012 Oct;46:107-15. doi: http://dx.doi.org/10.1590/S0080-62342012000700016

13. Cunha JB, Dutra RA, Salomé GM, Ferreira LM. Computational system applied to mobile technology for evaluation and treatment of wounds. J Nurs UFPE Online. [Internet]. 2018 May [cited Sep 10, 2019];12:1263-72. Available from: https://periodicos.ufpe.br/revistas/revistaenfermagem/article/view/230677/30457

14. Cunha DR, Salomé GM, Massahud Junior MR, Mendes B, Ferreira LM. Development and validation of an algorithm for laser application in wound treatment. Rev Lat Am Enfermagem. 2017 Dec;25:e2955. doi: http://dx.doi. org/10.1590/1518-8345.1998.2955

15. Santos AC, Dutra RA, Salomé GM, Ferreira LM. Construction and internal reliability of an algorithm for choice cleaning and topical therapy on wounds. J Nurs UFPE Online. [Internet]. 2018 May [cited Sep 10, 2019];12:125062. Available from: https://periodicos.ufpe.br/revistas/revistaenfermagem/article/view/230675/28869

16. Webb R. The 3D future of wound healing. J Wound Care. 2016 Oct;25:559. doi: http://dx.doi.org/10.12968/ jowc.2016.25.10.559

17. Rezende LC, Santos SR, Medeiros AL. Assessment of a prototype for the Systemization of Nursing Care on a mobile device. Rev Lat Am Enfermagem. 2016 Jul;24:e2714. doi: http://dx.doi.org/10.1590/1518-8345.0898.2714

18. De Lima ED, Fleck CS, Borges JJ, Condessa RL, Vieira SR. Effects of educational intervention on adherence to the technical recommendations for tracheobronchial aspiration in patients admitted to an intensive care unit. Rev Bras Ter Intensiva. 2013 Apr-Jun;25:115-22. doi: http://dx.doi.org/10.5935/0103-507X.20130022

19. Heck P, Klabbers M, van Eekelen M. A software product certification model. Software Qual J. [Internet]. 2010 Mar [cited Sep 10, 2019];18:37-55. Available from: https://link.springer.com/article/10.1007/s11219-009-9080-0

20. Silva AC, Bongiolo MR, Toscan S, Silva Junior AF, Bo K, Koch KS, et al. The impact of respiratory physical therapy and endotracheal suctioning in preterm newborns in the first week of life. Rev AMRIGS. [Internet]. 2014 Jul 
[cited Sep 10, 2019];58:213-9. Available from: http://ivrozilla.com/doc/915558/07_1381_revista-amrigs.indd

21. Tibes CM, Dias JD, Zem-Mascarenhas SH. Mobile applications developed for the health sector in Brazil: an integrative literature review. Rev Min Enferm. [Internet]. 2014 Apr-Jun [cited Sep 10, 2019];18:471-86. Available from: http://reme.org.br/exportar-pdf/940/en_v18n2a16.pdf

22. VêscoviSJ, Primo CC, Sant'AnnaHC, Bringuete ME, RohrRV, PradoTN, et. al. Mobile application for evaluation of feet in people with diabetes mellitus. Acta Paul Enferm. [Internet]. 2017 Nov-Dec [cited Sep 10, 2019];30:607-13. Available from: http://www.scielo.br/scielo.php?script=sci_arttext\&pid=S0103=21002017000600607-\&lng=en\&nrm=iso\&tlngen

23. Oliveira TR, Costa FMR. Development of mobile application for reference to vaccination in Brazil. J Health Inform. [Internet]. 2012 Jan-May [cited Sep 10, 2019];4:23-7. Available from: http://www.jhi-sbis.saude.ws/ojs-jhi/index. php/jhi-sbis/article/view/161/109 